\title{
Detection of Unknown Crypts under the Floor in the Holy Trinity Church (Dominican Monastery) in Krakow, Poland
}

\author{
Anna Strzępowicz ${ }^{1,}$, Mikołaj Łyskowski ${ }^{2}$, Jerzy Ziętek $^{1}$, and Sylwia Tomecka-Suchon ${ }^{1}$ \\ ${ }^{1}$ AGH University of Science and Technology, Faculty of Geology, Geophysics and Environmental \\ Protection, A. Mickiewicza Av. 30, 30-059 Krakow, Poland \\ ${ }^{2}$ AGH University of Science and Technology, University Computer Centre, A. Mickiewicza Av. 30, \\ 30-059 Krakow, Poland
}

\begin{abstract}
The GPR surveying method belongs to non-invasive and quick geophysical methods, applied also in archaeological prospection. It allows for detecting archaeological artefacts buried under historical layers, and also those which can be found within buildings of historical value. Most commonly, just as in this particular case, it is used in churches, where other non-invasive localisation methods cannot be applied. In a majority of cases, surveys bring about highly positive results, enabling the site and size of a specific object to be indicated. A good example are the results obtained from the measurements carried out in the Basilica of Holy Trinity, belonging to the Dominican Monastery in Krakow. They allowed for confirming the location of the already existing crypts and for indicating so-far unidentified objects. Key words - GPR, crypts, Basilica of Holy Trinity, Krakow.
\end{abstract}

\section{Introduction}

For many years, ground penetrating radar (GPR) surveys serve as a tool for investigation of historical complexes such as: churches, basilicas or ancient settlements. In most cases it is closely related to the specifics of place - e.g. churches are place of worship, and also a place of burial for the monks, people of merit or with a high social status. Among numerous historic buildings which can be found in Krakow, the Dominican Monastery is one of the oldest ones. The origins of this architectural object date back to the 13th century, i.e. the period of economic and cultural growth in Krakow [1]. Inter alia, the facilities belonging to the Dominican Monastery complex were taken into account when the water supply and sewage disposal system of the city, future medieval capital of Poland, was created. Despite the importance of the Church at that time, there were disputes between the city and the Dominicans which would continue until the 17th century. They referred to the most crucial issue - access to clean drinking water, as well as to water that would drive water mills [2]. The Basilica of Holy Trinity, belonging to the Dominican Monastery, originally had a form of a three-nave hall in the redbrick Gothic style. At the turn of the 14th and 15th centuries it

\footnotetext{
*Corresponding author: tomecka@agh.edu.pl
} 
was converted into the basilica style. Until half of the 19th century, one of the most characteristic elements of the exterior of the temple was a stone bell tower, separated from the main building and erecting in front of its facade at the end of Stolarska Street. However, after the fire of 1850, the only remains of the church bell tower were walls blackened by smoke. They were demolished in the course of reconstruction works, and in 1876 a neo-Gothic porch was added to the facade of the temple. It sheltered a 14th century Gothic portal of the main entrance, which was renovated in 1893 [3].

Investigations, initiated by administration employee and archaeologists, were aimed at checking if as then undiscovered crypts, other burial rites or other important archaeological objects could exist under the floor of this historic church. These works were supposed to be carried out before the collision-free installation of a heating system or other elements of the infrastructure included in the design.

The GPR method, selected for this purpose, is one for the basic tools for this kind of research $[4,5]$ and it was the main reason for its implementation in this case study. Application of this survey type is very popular in Poland. The main advantage of the GPR method is its totally non-destructive nature which allows to perform measurements even within the most buildings, especially those with cultural and historical biggest value. Moreover, considering the characteristics of the site and physical working space (which was limited), the method chosen by the authors was more than adequate.

\section{Method}

The GPR method belongs to non-invasive, high resolution, and fast application geophysical survey. Sometimes in undergoing measurements, one is capable of identifying locations of anomalous areas at the very early stage such as data collection. This method has applications in various fields, one of them is archaeology. The basic measuring equipment consists of a central unit and two antennae: a transmitting and receiving one. The first one, the transmitting antenna, emits short pulses of electromagnetic waves, which are subject to reflection, refraction and attenuation when propagating. The most important phenomenon from the point of view of the GPR method is the reflection of the wave on the interface of two media characterised by different physical parameters, which occurs in the case of the contrast between the dielectric constants of these media. The value of the dielectric constant has a major impact on the velocity of electromagnetic wave propagation in a medium. The reflected wave is recorded by the receiving antenna. The obtained wave image reflects the structure of the medium. The depth range of the GPR method usually does not exceed the distance of several meters, and the depth of penetration heavily depends on the structure of the examined medium, the frequency of the emitted electromagnetic wave and the humidity of the soil. It also depends on the pre-set recording parameters. It is generally assumed that for antennae of $500 \mathrm{MHz}$ frequency, the depth range is $6 \mathrm{~m}$ on average. It is also important to take the resolution parameter into account, as it increases with an increase of the antenna frequency, which deteriorates the penetration capability of the antenna [6, 7].

It has to be emphasised that the quality of obtained results strongly depends on the optimal selection of measurement parameters such as: the sampling frequency of the input signal, the number of samples, the stacking and the distance between particular traces [8].

The equipment used in the measurements was a Mala GeoScience GPR with a $500 \mathrm{MHz}$ shielded antenna. Distance on the profile was measured with a calibrated mechanism calculating the number of revolutions into meters. 


\section{Raw data processing}

In total, there were 62 measured profiles. The entire investigated area was divided into subareas: "Main transept", "Right nave", "Left nave", "Crosswise", "Presbytery", "Vestry", "Altar" and "Behind altar".

In order to adjust the range of the depth scale, the velocity of wave propagation of $0.1 \mathrm{~m} / \mathrm{ns}$ was adopted for the purposes of the time-depth conversion. The depth scale was assumed on the basis of specified propagation velocity of the electromagnetic wave in the studied medium [9]. The value of the velocity changes form $0.3 \mathrm{~m} / \mathrm{ns}$ (in the air) to $0.03 \mathrm{~m} / \mathrm{ns}$ (in the fresh water) [7]. It was impossible to perform the WARR (Wide-Angle Reflection and Refraction) velocity profile. The permissible error in adjusting the velocity is $0.01 \mathrm{~m} / \mathrm{ns}$.

Prior to the presentation of the results, the raw data were processed, so they were subjected to an increase in the signal-to-noise ratio. The data used in this process were subjected, inter alia, to [10]:

- static adjustments, the aim of which is to move the first penetration to 0 on the depth scale (move starttime), or to cut off the lower part of information which includes the noise (time cut);

- procedures bringing the average value of the amplitude to zero (subtract-DC-shift);

- eliminate low frequency signal disturbance (subtract-mean (dewow));

- frequency filtering aimed at the elimination of the noise (bandpassbutterworth);

- enhancement, increasing the value of the amplitude weakened due to the electromagnetic wave attenuation in the investigated medium (gain function);

- smoothening, the aim of which is eliminating single noises by means of the averaging of the amplitude value, e.g. in the time-distance window (median $x y$-filter);

- remove horizontal ringing from signal traces (background removal).

All above processing procedures allowed for acquiring very good quality data for interpretation.

\section{Results and discussion}

From among all the profiles, the profile 5 from the areas labelled as "Left Nave" and "Right Nave" and the profile 9 from the "Altar" area were selected for presentation. The profile across the area called "Vestry" seemed to be interesting for the authors, therefore the profiles 4 and 16 from the area will also be presented. Also interesting seems to be profile 19 in the "Vestry" area, on which anomalies form construction elements can be observed.

The interpretation of the results is presented on the enclosed map (Fig. 1), on which the sites where significant anomalies were spotted are marked. The areas marked with the letter "C" are connected with the occurrence of crypts under the church floor. The remaining sites do not allow for assuming any definitive conclusions.

On the below figure (Fig. 2), in the "Left Nave" area, on the profiles 2 to 7 there is a clearly distinguished anomalous zone, probably resulting from the presence of a crypt of a larger size. At the ends of the profiles (from the distance of approx. 30-32 running meters means that this in the distance from the beginning of the profile), an anomaly at a larger depth (approx. 2-2.5 m) can be seen. The possibility that it is caused by a crypt cannot be excluded. The perpendicular profile 3 - "Crosswise" area, shows strong anomalous zone between 14 and 20 running meters, correlating with that seen on profile 5. Similarly, in the "Right Nave" area, on the profiles 6, 5, 1, 2 and 3 significant anomalies can be observed, which is most probably caused by the existence of crypts under the floor. Anomalies between 2 and 6 running meters on "Crosswise" profile 10 correlates with that seen on profile 5 in the "Right Nave" subarea. Also anomalies which can be seen at the ends of the 
profiles 5 and 6 may be due to the existence of a crypt. The presence of a crypt on the profile 4 is confirmed by the "Crosswise" profiles 1,2 and 3. What was innovative here was the instantaneous verification of the anomalies by archaeologists by means of drilling small-diameter boreholes in the floor.

The main aim of conducting the perpendicular profiles was to confirm the occurrence of the earlier discovered anomalies. They confirmed all the anomalous areas that had been indicated before. As it has already been mentioned, they allowed for the identification of other crypts as well. It was at the beginning of the profiles 1, 2 and 3 that such a site was indicated at the threshold of the chapel.

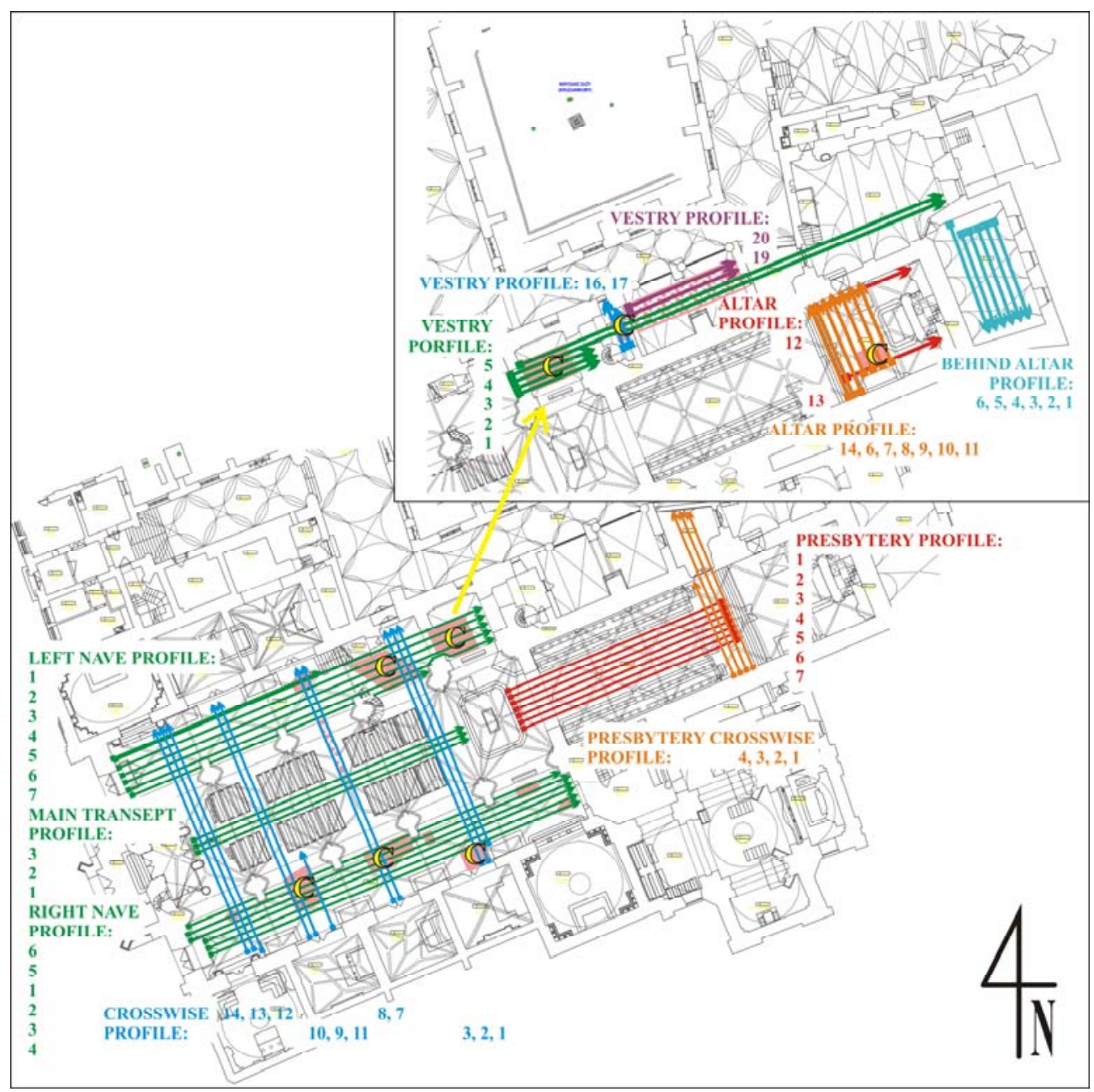

Fig. 1. Schematic map of the Basilica of Holy Trinity (non scaled) with drawn GPR profiles and anomalous area marked (C - crypts) 
Left Nave profile 5

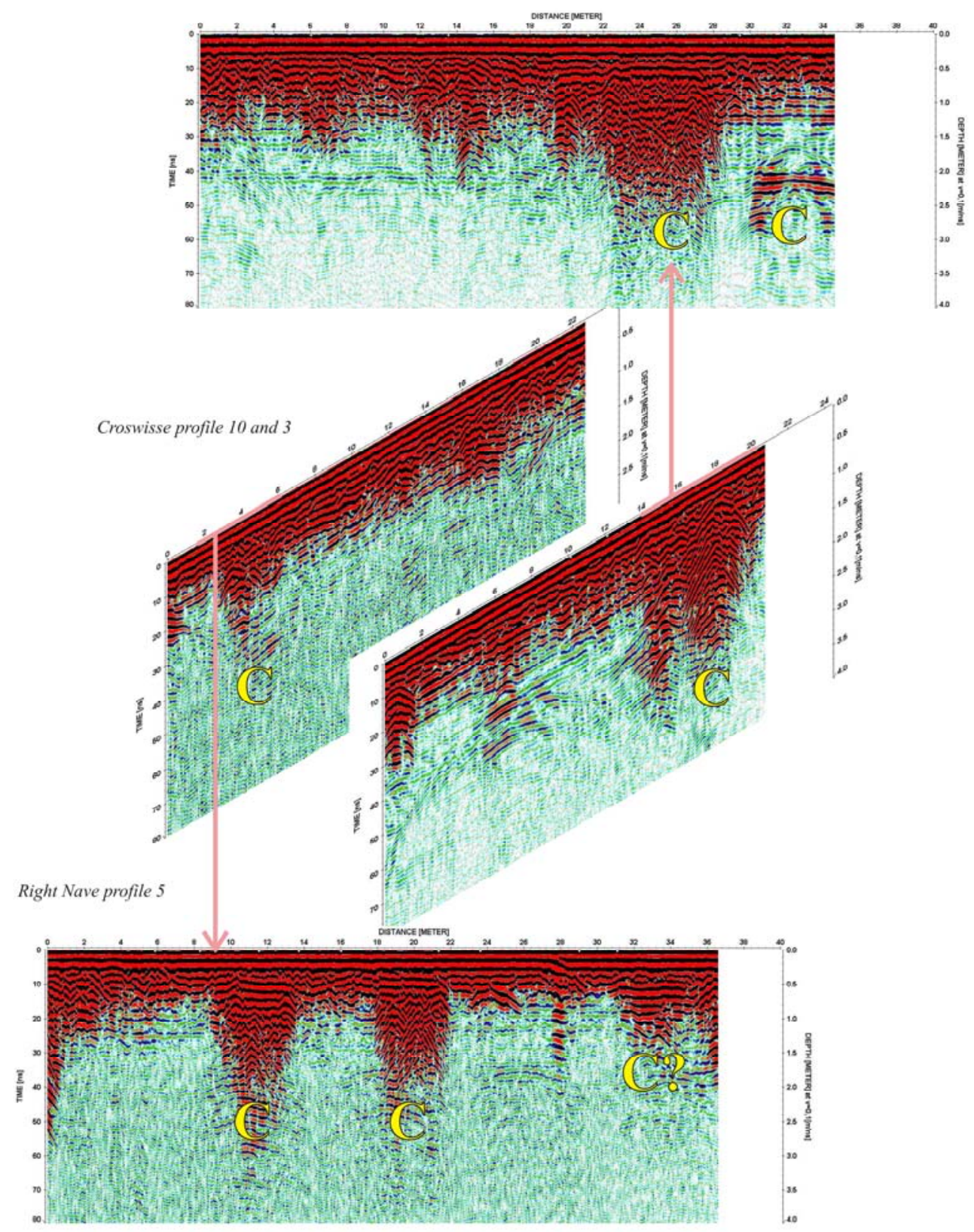

Fig. 2. Processed images from GPR profiles 5 made in the "Left Nave" and "Right Nave" subarea, and perpendicular profiles 10 and 3 from the "Crosswise" subarea. "C" indicates anomalous zone detected crypt

The above image (Fig. 3) obtained from the profile 9 indicates an anomaly, between 0 and $2 \mathrm{~m}$ and at a depth of $1 \mathrm{~m}$, which is very similar to the anomalies recorded above the crypts whose existence had been confirmed. It can be seen at a small depth of $0.3-0.5 \mathrm{~m}$ at the beginning of the profile. The perpendicular profile 13 shows strong reflections between 3 and 7 running meters, the origin of which is a crypt parallel to the profile. 




Fig. 3. Processed images from GPR profiles 9 and 13 made in the "Altar" subarea. "C" indicates anomalous zone - detected crypt

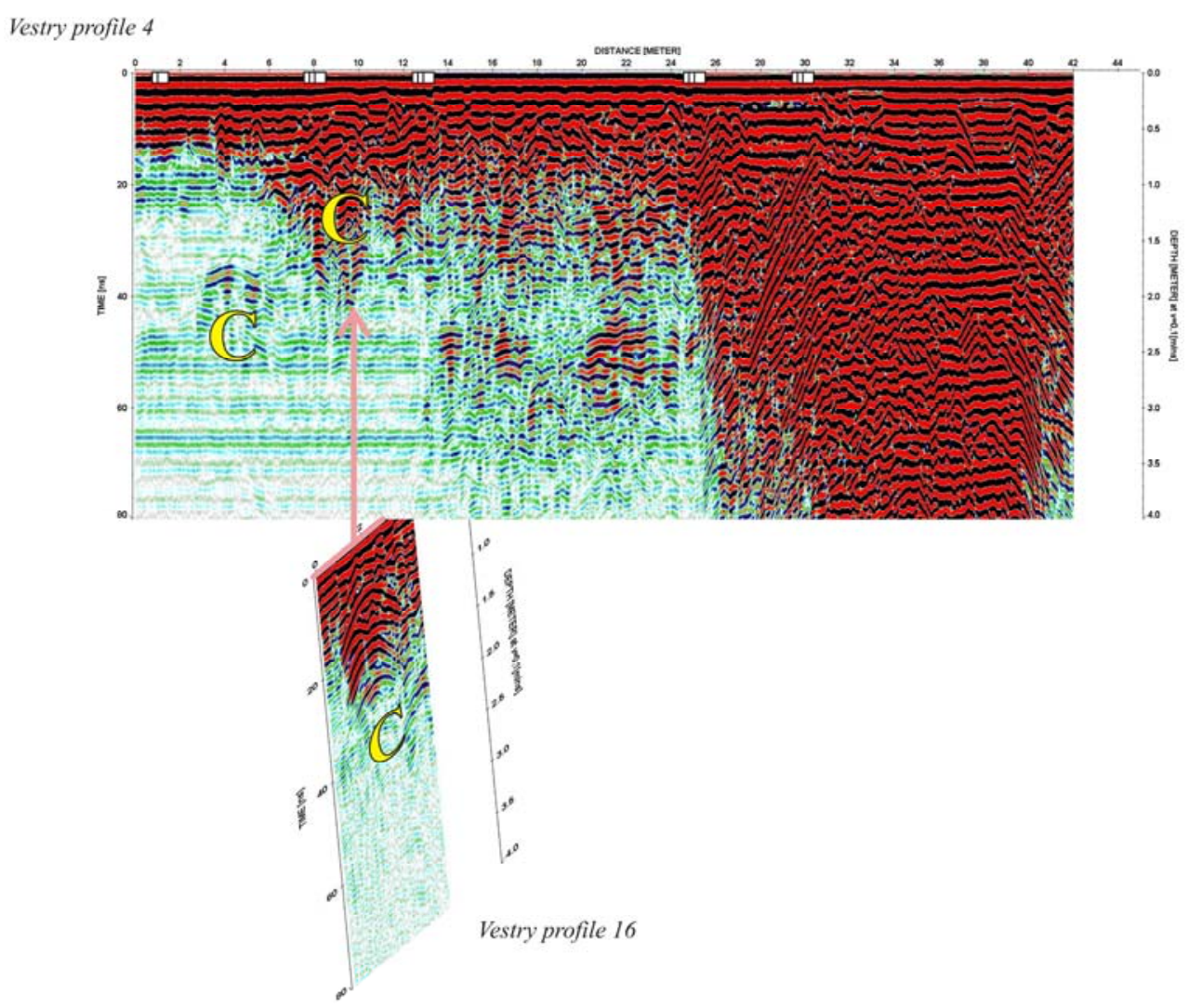

Fig. 4. Processed images from GPR profiles 4 and 16 made in the "Vestry" subarea. "C" indicates anomalous zone - detected crypt

Multiple markers on the horizontal axis indicate the passage through a door (Fig. 4). The anomalies recorded at the ends of the profiles 2 to 6 in the "Left Nave" can be observed also in the profiles 1 to 5 in the "Vestry" region. Vertical boundaries of these anomalies 
indicate the existence of an anthropogenic object which could be a crypt. On the profiles 1 to 4 it is located between 3 and 6 running meters. On the profile 5 , which is shorter, it occurs before 3 running meters. Compared to other anomalies confirmed by the drilled boreholes, it is situated significantly deeper. On all the profiles running across the "Vestry" (profiles 4, 5, 19, 20) anomalies starting at floor beams and at a depth of approximately 2.3 $\mathrm{m}$ can be seen. At the end of shown profile 4 form "Vestry" subarea - between 25 and 40 running meters, high amplitude anomalous area is visible. Its source is connected with room in the basement.

Additional profiles 16 and 17 were performed in order to verify the anomalies recorded on the profiles parallel to the main axis of the church (4 and 5). These are the profiles where a clearly visible shallow anomaly can be observed, which unambiguously indicates the existence of a crypt. The ceiling of this crypt is probably at a depth of $0.5 \mathrm{~m}$.

During the measurements there was also possible to identify anomalies caused by construction beams in the floor in the "Vestry" subarea. They are visible at depth $0.25 \mathrm{~m}$ on above image (Fig. 5) small hyperbolas (indicated by arrows).

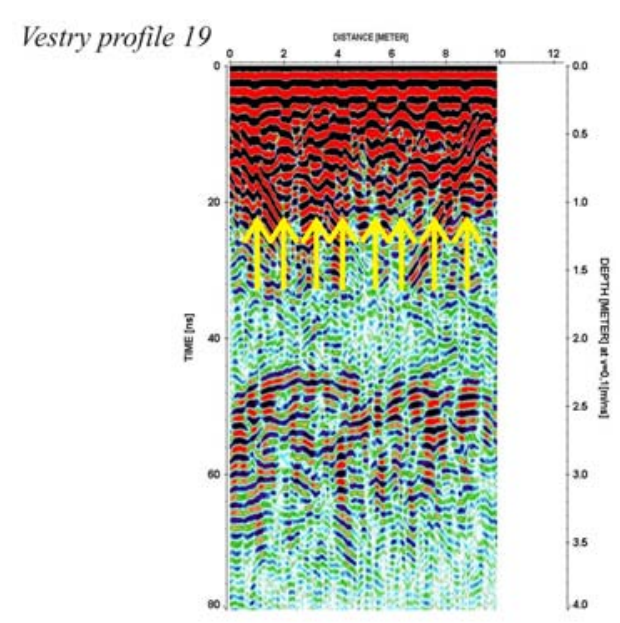

Fig. 5. Processed image from GPR profiles 19 "Vestry" subarea showing construction beams (marked by arrows)

\section{Conclusion}

Collected and processed data allowed interpretation of image up to $3 \mathrm{~m}$ in depth. The appropriately selected surveying parameters and proper methodology of these measurements was adequate to expected results. In this case study it was possible to perform instantaneous localisation and even verification of the results.

The discovered and identified anomalies which are due to the existence of crypts allow for designing a collision-free layout of a heating system or other installations in accordance with the planned concept. It was thanks to it that completely non-invasive, quick and highresolution GPR measurements brought about high-quality results.

Considering the characteristics of the site and physical working space, the method chosen by the authors was more than adequate. In this case, the field experience of the authors [11] was crucial for the quality of obtained results. Authors previously conducted similar type of research in other churches [12]. In their opinion similar result could be obtained with use of shielded antennae with 250 and even $800 \mathrm{MHz}$ frequency. 


\section{References}

1. Z. Kozłowska, Rocznik Krakowski 20, 1-19 (1926)

2. U. Sowina, Woda i ludzie w mieście późnośredniowiecznym i wczesnośredniowiecznym, 76-104 (2009)

3. J. Muczkowski, Rocznik Krakowski 20, 40-56 (1926)

4. J. Panisova, M. Frastia, T. Wunderlich, R. Pasteka, D. Kusnirak, Archaeological Prospection 20, 163-174 (2013)

5. L.B. Conyers, Interpreting Ground-penetrating Radar for Archaeology (2012)

6. J.M. Reynolds, An Introduction to Applied and Environmental Geophysics, 2nd Edition, 289-348, 535-624 (2011)

7. A.P. Annan A.P., Ground Penetrating Radar Workshop Notes (2001)

8. Ł. Ortyl, J. Karczewski, E. Mazurkiewicz, SGEM 2017: 17th international multidisciplinary scientific geoconference: science and technologies in geology, exploration and mining: 29 June-5 July, 2017, Albena, Bulgaria: conference proceedings 17(14), 173-180 (2017)

9. M. Łyskowski, E. Mazurek, Logistyka 4, 330-336 (2013)

10. K.J. Sandmeier, ReflexW (2009)

11. J. Ziętek, K. Wawrzyniak-Guz, E. Mazurkiewicz, M. Łyskowski, Nawarstwienia historyczne miast Europy Środkowej, 397-405 (2016)

12. A. Strzępowicz, J. Ziętek, VIET-POL 2015: Second International Conference on Scientific Research Cooperation Between Vietnam and Poland in Earth Sciences, 117-132 (2015) 